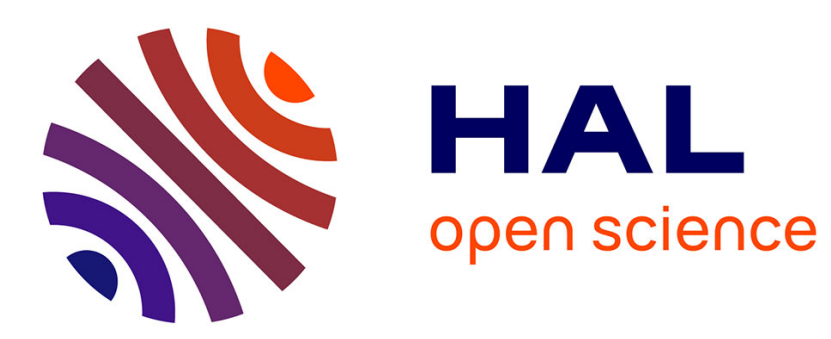

\title{
Corps rebelles: la mode des jeunes urbains dans les années 1960-1970 au Mali
}

Ophélie Rillon

\section{To cite this version:}

Ophélie Rillon. Corps rebelles: la mode des jeunes urbains dans les années 1960-1970 au Mali. Genèses. Sciences sociales et histoire, 2010, 81 (4), pp.64-83. 10.3917/gen.081.0064 . hal-01540041

\section{HAL Id: hal-01540041 \\ https://hal.science/hal-01540041}

Submitted on 15 Jun 2017

HAL is a multi-disciplinary open access archive for the deposit and dissemination of scientific research documents, whether they are published or not. The documents may come from teaching and research institutions in France or abroad, or from public or private research centers.
L'archive ouverte pluridisciplinaire HAL, est destinée au dépôt et à la diffusion de documents scientifiques de niveau recherche, publiés ou non, émanant des établissements d'enseignement et de recherche français ou étrangers, des laboratoires publics ou privés. 


\title{
CORPS REBELLES : LA MODE DES JEUNES URBAINS DANS LES ANNÉES 1960-1970 AU MALI
}

\author{
Ophélie Rillon
}

Belin | «enèses »

2010/4 $n^{\circ} 81 \mid$ pages 64 à 83

ISSN 1155-3219

ISBN 9782701156682

Article disponible en ligne à l'adresse :

http://www.cairn.info/revue-geneses-2010-4-page-64.htm

\section{Pour citer cet article :}

Ophélie Rillon, « Corps rebelles : la mode des jeunes urbains dans les années 1960-1970 au Mali », Genèses 2010/4 (n 81), p. 64-83.

DOI 10.3917/gen.081.0064

Distribution électronique Cairn.info pour Belin.

(C) Belin. Tous droits réservés pour tous pays.

La reproduction ou représentation de cet article, notamment par photocopie, n'est autorisée que dans les limites des conditions générales d'utilisation du site ou, le cas échéant, des conditions générales de la licence souscrite par votre établissement. Toute autre reproduction ou représentation, en tout ou partie, sous quelque forme et de quelque manière que ce soit, est interdite sauf accord préalable et écrit de l'éditeur, en dehors des cas prévus par la législation en vigueur en France. Il est précisé que son stockage dans une base de données est également interdit. 


\section{$\approx$ Corps rebelles: la mode des jeunes urbains dans les années 1960-1970 au Mali*

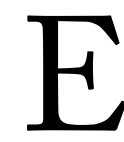

n 1960, lorsque le Mali accéda à l'indépendance, les dirigeants politiques conduits par Modibo Keita firent le choix du socialisme comme fondement de la Construction nationale. Parades militaires et défilés populaires étaient l'occasion de mettre en scène les forces vives de la nation dont la jeunesse militante constituait «l'avant-garde», en tant que «fer de lance» de la révolution ${ }^{1}$. Les préoccupations étatiques se portèrent également sur les corps féminins qui incarnaient le lieu de production et de reproduction des nouveaux citoyens masculins devant émerger de la société socialiste:

«Nos sœurs [...] sont à l'honneur et méritent l'appellation de militantes conscientes et combattantes de la révolution, non seulement parce qu'elles sont guidées par le souci d'avoir des enfants sains et robustes mais aussi et surtout par celui de préparer des hommes qui, demain, assureront la continuité d'un Mali en perpétuel progrès ${ }^{2} »$.

Drapées de boubous à l'effigie de Modibo Keita et de la production nationale malienne - mangues, arachides - coiffées de tresses baptisées "Année 1960 », «Nation», «Négritude» et de foulards «Bonjour mon Président», les corps des femmes en âge d'être mariées et mères furent des supports de diffusion de la propagande socialiste (Ayina 1987).

Dans un contexte de guerre froide et de libération nationale, tout ce qui venait des puissances impériales, notamment de la France, ancienne métropole, et des États-Unis, symbole du capitalisme, était à bannir aux yeux des dirigeants maliens. Seules les références aux pays dits socialistes, aux mouvements panafricaniste et tiers-mondiste, étaient acceptées sur le plan culturel. Pourtant, une partie de la jeunesse malienne avait les yeux rivés vers l'extérieur. Influencés par le cinéma et les magazines comme Salut les copains, Elles, Intimités, les jeunes 
Bamakois s'approprièrent différentes «cultures globales de la jeunesse» (Comaroff et Comaroff 2000: 94) issues des pays du Nord33. Ils furent yéyé, twist, hippie, rock, pop au gré des époques et des tendances. Plus qu'un mimétisme, une réinvention culturelle eut lieu dans la mode vestimentaire, les musiques, les danses. Les orchestres ${ }^{4}$, chargés de promouvoir la culture locale en puisant dans les répertoires régionaux, acquéraient leur célébrité en intégrant les rythmes jazz et les sons électrifiés qui faisaient danser la jeunesse urbaine. Boubacar Traoré, avec son tube Mali Trwist, fut le symbole de ce cosmopolitisme des années 1960. Icône de la jeunesse malienne, vêtu d'un jean et d'une veste à franges, surnommé «Kar Kar, le Blouson noir», «l'Elvis Presley ou le Johnny Hallyday malien», il n'en était pas moins admiré par les soldats du régime socialiste qui lui faisaient le salut militaire lorsqu'ils le croisaient (Lieve 2002: 214). Les vêtements à la mode, robe courte, jupe gonflante, pantalon pattes d'éléphant, chemise serrée étaient réalisés à bon marché par les tailleurs du quartier comme Boubacar Traoré. Ainsi, le slogan de liberté scandé par les dirigeants résonnait dans l'esprit des jeunes comme une pratique quotidienne. Comme l'exprimait Edgar Morin au sujet de la mode yéyé en France, cette culture jeune «recouvrait non seulement un domaine musical, mais une certaine façon d'être, presque une attitude envers la vie» (1969: 766). Les cadets sociaux (Bayart 1979: 233-281) revendiquaient une autonomie, une identité, une existence propre qu'ils symbolisèrent par l'appropriation et la réinvention d'une mode venue de l'extérieur. Si la mode des années 1960 se plaçait sous le signe de la révolution ${ }^{5}$ (Veillon, Guillaume et Ruffat 2000; Hobsbawm 1999: 428-431; Poiger 2000: 333), il s'agit ici d'en étudier la teneur contestataire dans le contexte malien. Bien entendu, la jeunesse malienne ne fut pas imprégnée dans son ensemble de cette influence extérieure. Seule la jeunesse urbaine pouvait avoir accès aux magazines, disques vinyles et autres biens de consommation européens qui transitaient par les pays limitrophes comme le Sénégal ou la Côte-d'Ivoire6, le Congo-Kinshasa (Gondola 1997), le Congo-Brazzaville (Gandoulou 1989: 239) ou les pays anglophones tels que le Ghana ou le Libéria, marqués par le mouvement afro-américain. Mais la jeunesse rurale n'était pas non plus totalement hermétique à cette mode: les migrations régionales saisonnières, l'exode rural en furent aussi des vecteurs de diffusion dans des espaces plus reculés 7 .

Les photographies réalisées par Malick Sidibé dans les années 1960-1970 permettent d'appréhender cette nouvelle culture jeune. En 1962, le photographe malien ouvrit son studio à Bagadadji, quartier central et populaire de Bamako. Comme il le dit lui-même, il fut «le seul jeune reporter de Bamako à faire des photos dans les surprises-parties, les «surpat'» (Magnin 1998: 36). Il fut le photographe des corps en mouvements de la jeunesse urbaine de Bamako jusqu'en 1976, période où commencèrent à disparaitre les clubs de jeunes et où il réintégra définitivement son studio. Dans ses photographies de studio, Malick Sidibé dirigeait les corps de ses sujets, choisissant leurs positions et parfois même les vêtements et accessoires qu'ils devaient porter, alors qu'au cours de ses reportages, c'étaient les 
jeunes eux-mêmes qui se mettaient en scène. Au final pourtant, ces deux types de photographies sont très proches car ils sont le reflet d'une intégration de codes communs. S’il était un peu plus âgé que ses sujets, Malick Sidibé intégra à leur contact les référents musicaux, vestimentaires et cinématographiques qui baignaient leurs imaginaires et les représentations qu'ils avaient d'eux-mêmes. D'autre part, ces jeunes étaient ses clients; il se devait donc de révéler leurs rêves et leurs identifications: "celui que je me crois, celui que je voudrais qu'on me croie, celui que le photographe me croit et celui dont il se sert pour exhiber son art», comme l'écrit Roland Barthes (2009: 29). Cet article utilise un ouvrage (Magnin 1998) qui rassemble des clichés de Malick Sidibé et des témoignages d'anciens «ambianceurs». Ce corpus est le reflet d'un «âge d'or» de la photographie malienne (Nimis 2005: 390) qui, pour reprendre les mots de Malick Sidibé, visait à «embellir la vie» (Werner 1996: 315). Ces photographies doivent donc être appréhendées comme des traces, des indices relevant d'une reconstitution sélective d'un passé, aujourd'hui largement occulté dans les mémoires maliennes ${ }^{8}$ et l'historiographie. La mode des jeunes urbains influencée par l'étranger étant très mal vue des autorités, c'est dans les archives de la répression et de la propagande qu'il en est question: archives du Bureau politique national de l'US-RDA 9 , archives de police et de gendarmerie, colonnes du journal gouvernemental L'Essor, seul autorisé à l'époque. Dans ce contexte de «surchauffe politique» (Mbembe 1988:143), ces documents témoignent d'un discours répressif à l'égard des jeunes, qui emprunte à la fois aux représentations sexuées coloniales (Goerg 2007) et à celles véhiculées par les régimes autoritaires, soviétique ou maoïste (Fitzpatrick 2006). Au-delà du discours de propagande et des pratiques de répression, l'ensemble de ces sources permet d'appréhender la teneur transgressive du "yéyéisme» 10 , et l'attitude de l'État à l'égard de cette mode, oscillant entre indifférence, étouffement ou répression violente selon les contextes.

Cette étude vise donc à analyser les pratiques corporelles des jeunes urbains, définies par leurs attributs (vêtements, coiffures, accessoires) et leurs attitudes (postures, mimiques, danses), comme un «langage politique» (Allman 2004 : 247). Celui-ci ne se situerait pas dans une confrontation directe à l'État, mais bien plus dans un contournement du projet politique autoritaire et des normes sociales qui en découlaient. Dans les premières années de l'indépendance (19601965), les jeunes urbains établirent de nouveaux codes qui défiaient les normes sociales édictées par l'État socialiste. D'abord indifférent à cette culture juvénile, l'État se radicalisa à partir de 1966 et déclencha une vaste campagne de moralisation de la société. L'objectif affiché, on le verra, était la reprise en main des corps pour obtenir un assainissement des esprits propice à l'avènement du socialisme scientifique. En 1968, un groupe de jeunes militaires renversa le régime socialiste et mit fin à ses dérives autoritaires. Symbole d'une liberté retrouvée, les jeunes réinvestirent leurs corps, cette fois-ci au travers d'une mode bien plus politique, où les jeunes filles occupèrent une place plus marginale. 
Les indépendances: construction nationale et transgression des normes corporelles (1960-1965) sexe, ou d'origine sociale et géographique, en de multiples structures fondées sur l'idée du "soldat citoyen» qui associaient formation civique et physique. Les deux grandes structures de masse de la jeunesse furent le Mouvement national des pionniers et les Brigades de vigilance, mises en sommeil à partir de 1964 au profit des milices populaires. Symbole de la militarisation de la société, chaque organisation disposait de son uniforme, dont le plus significatif était celui des miliciennes qui, comme leurs homologues masculins, portaient au quotidien une tenue militaire mais défilaient en boubous et en armes lors des cérémonies officielles (voir document p. 68).

$\mathrm{Si}$, dans leurs discours, les dirigeants politiques distinguaient les rôles sociaux des hommes et des femmes, l'idée selon laquelle une «bonne ménagère » devait être, avant tout, une «bonne citoyenne» eut des conséquences inattendues: hommes et femmes intégrèrent les mêmes structures et leurs traitements étaient à peu près équivalents. Jusqu'à l'âge de quinze ans - âge légal du mariage pour les jeunes filles - la mixité prévalait, puis les deux sexes étaient séparés mais pour suivre une formation similaire. Suivant l'idéologie du régime, le sport y occupait une place importante: la formation d'esprits sains passait par des corps robustes et disciplinés, charpentés par les activités sportives, de plein air et les chantiers de construction imposés dans ces organisations. Avançant la tête haute, dans une gestuelle commune - démarche élancée, bras le long du corps - et vêtus d'uniformes, ces groupes de jeunes hommes et/ou de jeunes femmes se faisaient l'écho d'une construction nationale en marche. Sans s'en rendre compte, le gouvernement de Modibo Keita avait ouvert la porte à une modification des représentations sexuées: des jeunes femmes avaient incorporé des normes considérées jusque-là comme masculines. Elles étaient entraînées et vêtues comme les hommes. Elles portaient des uniformes, des pantalons, des armes.

Néanmoins, cette uniformisation des corps masculins et féminins fut largement critiquée par la population. Les normes sociales promues par l'État socialiste, dans son souci égalitariste, bouleversaient l'ordre gérontocratique et patriarcal11. Les parents virent d'un très mauvais œil la mixité instaurée dans les organisations de jeunesse et sur les bancs de l'école: «le mouvement [pionnier] c'est fait pour engrosser nos enfants", disait-on (Nédélec 1994: 335). Certains maris se plaignirent à la Commission sociale des femmes que leurs épouses miliciennes «imaginent que le port du pantalon les dispense de leurs devoirs conjugaux ${ }^{12}$. Enfin, la jeunesse urbaine et scolarisée, qui avait constitué les premiers éléments de ces organisations, se détourna rapidement de ces structures parami- 


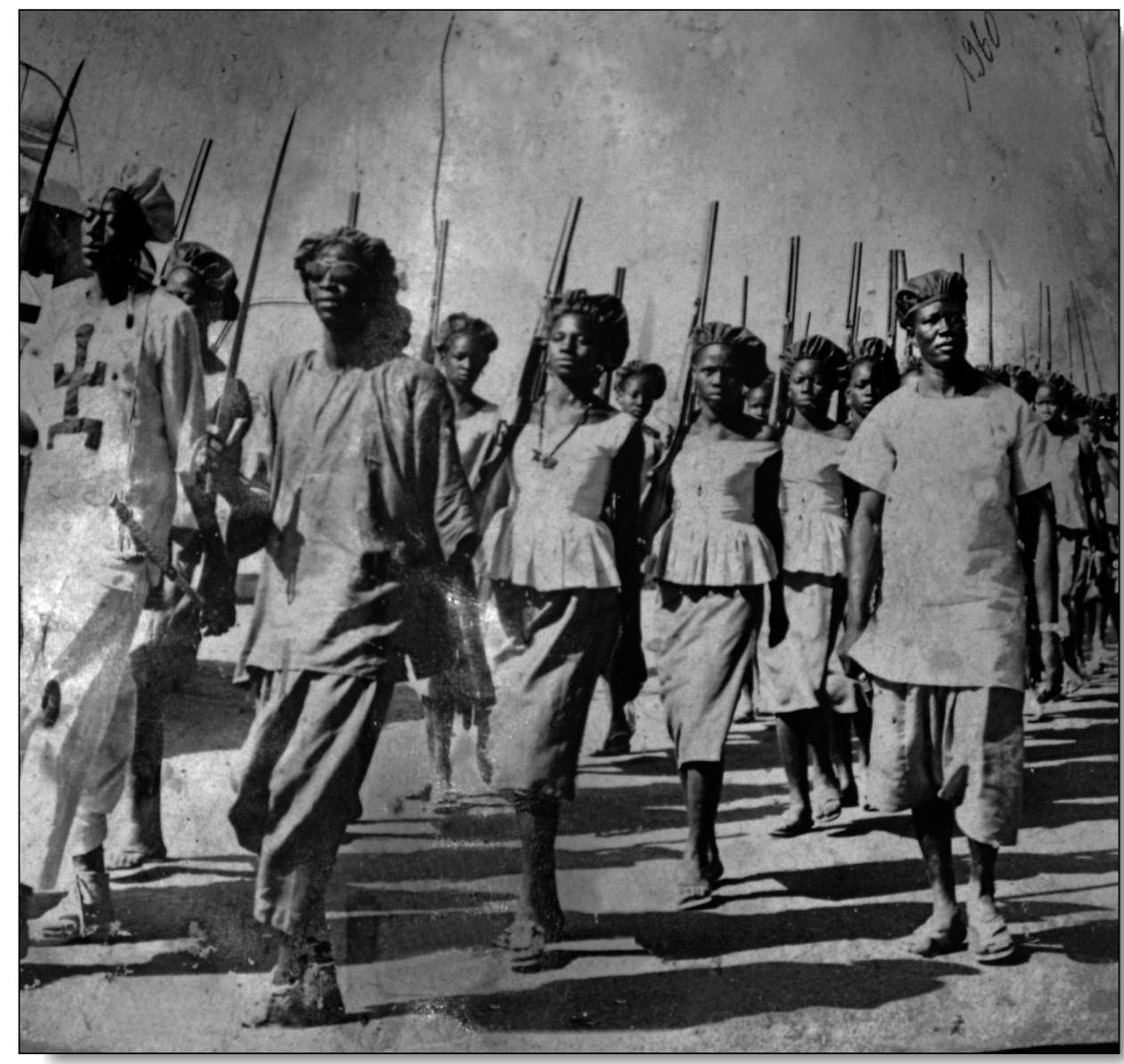

Document: Anonyme. « 1960 ». Affichée en février 2010 dans la salle d'archives de la mairie de Mopti.

litaires qu'elle jugeait trop autoritaires. De surcroît, si le projet socialiste plaçait les jeunes au cœur de sa politique économique et nationaliste, il ne cessait pour autant de les maintenir dans une position subalterne face aux aînés (ibid.: 331338) ${ }^{13}$. Contournant les espaces de sociabilité imposés par le régime, nombre de jeunes développèrent en marge leurs propres réseaux et loisirs (Diouf 2003).

\section{La célébration des corps: s'babiller pour être}

Dans les années 1950, les jeunes Soudanais dits «évolués»14 s'étaient approprié la mode des colonisateurs. Les costumes, les tailleurs, les chaussures à talon ainsi que les danses à deux - valses, cha cha cha - étaient entrés dans le quotidien de la minorité que constituait l'élite scolarisée. Comme l'explique Youssouf Doumbia, dit Garrincha, «ambianceur» de soirées15: 
«Dans les années 50, on prenait modèle sur les Zazous, en référence au groupe de Saint-Germain-des-Prés: des jeunes premiers nantis, fils de commerçants ou de fonctionnaires. Ils portaient la veste qui descendait jusqu'au genou, le pantalon audessus des mollets et des chaussettes de couleurs bien visibles. Ils prenaient des positions delta (jambes écartées et les deux mains tenant la veste), gamma (le buste jeté en arrière légèrement méprisant) ou lambda (à la Eddie Constantine). C'était un spectacle permanent, il fallait vivre à Bamako à cette époque pour le voir. Leurs pantalons étaient tellement moulants qu'ils ne pouvaient pas monter sur leur vélo. Ils marchaient à côté de leur vélo Peugeot, grand modèle, et se pavanaient au marché»

(Magnin 1998: 163).

Les jeunes femmes lettrées, encore plus minoritaires, n'échappaient pas non plus à cette mise en scène des corps:

«On monte à vélo, avec l'intention délibérée de choquer la société patriarcale, en balançant dans tous les sens le corps; on va jusqu'à défier la chronique coloniale en conduisant, comme c'est le cas de Marguerite Bertrand, une voiture»

(Konaré 1993: 54).

Les jeunes hommes engoncés dans leurs habits poussaient leurs vélos; les jeunes femmes ôtaient leurs foulards de tête, révélaient leurs coupes à la garçonne et, vêtues de robes ou de pantalons, elles enfourchaient ce moyen de locomotion masculin... les normes sexuées furent bousculées par une revendication d'autonomie et une quête d'individualité. Comme le montrèrent différentes études, l'école coloniale introduisit «de nouveaux modèles d'identité masculine et féminine» 16 (Barthélémy et Jézéquel 2007: 79) avec lesquels composèrent les élites lettrées, au gré de leurs intérêts personnels et des normes sociales en vigueur localement.

Mais ce fut surtout avec la Libération nationale que se démocratisa la libération des corps en milieu urbain. Si l'État socialiste tenta d'organiser l'ensemble de la société au sein de ses propres structures, il ne chercha pas pour autant - dans un premier temps - à dissoudre les "grins", ces réseaux sociaux de jeunes urbains tissés dans l'enfance, à l'école, dans le quartier, où se côtoyaient filles et garçons, tailleurs, vendeurs, petits commerçants, scolaires, et chauffeurs de taxi. Influencés par la culture occidentale, ces regroupements, appelés alors «clubs», portaient des noms inspirés des idoles, des films, des musiques du moment: Les Beatles, Les Copains, Al Capone, les Chats sauvages, Club Nouvelle Vague, etc. À chaque club correspondait un look, une façon de se comporter. Leurs membres se reconnaissaient à leurs tenues, leurs gestuelles, leur façon de parler et les clubs se faisaient une rude concurrence des apparences pour attirer à eux les filles. Penka Dembélé se rappelle cette codification minutieuse de la physionomie:

«la raie à gauche pour les célibataires et à droite pour les mariés [...] Il y a même un gars à Bamako qui se mettait tous les jours dans la peau de Buffalo Bill, mêmes gestes, mêmes attitudes. Pareil avec Bill Haley»

(Magnin 1998: 170).

Genèses 81, décembre 2010 
Rejetant les modèles corporels promus par le régime socialiste, cette mode s'affichait ouvertement contre l'africanisation des tenues (boubous, pagnes, foulards de tête). La mention "tenue correcte exigée», inscrite sur les cartons d'invitation aux «bals poussières», était plus spécifiquement destinée aux jeunes filles, moins influencées par cette occidentalisation vestimentaire ${ }^{17}$. Ainsi, certains clubs comme le Florina de Dravéla (Meillassoux 1968: 130-142) édictèrent des règles particulièrement strictes pour contrôler l'apparence de leurs membres féminins et les faire correspondre à leurs fantasmes. Il était interdit aux jeunes filles de se rendre aux soirées en «tenues africaines et en claquettes»: jupes courtes et talons hauts étaient recommandés. Pour intégrer ce club, elles devaient se plier aux critères de beauté occidentale - corps mince, taille de guêpe, maquillage. De nouvelles codifications furent introduites jusque dans les sphères les plus intimes des rapports sociaux: la monogamie fut imposée comme norme de couple et tout membre masculin surpris à propager des propos diffamatoires envers les conduites sexuelles d'une jeune fille était exclu. Dans un grand nombre de ces clubs, la galanterie fut érigée en règle d'or. Lors des soirées, un taxi venait chercher les jeunes filles devant chez elles et les ramenait à la maison. Petits et grands frères étaient chargés de les aider à se soustraire à l'autorité parentale. $\mathrm{Si}$, comme l'explique Erving Goffman (2002: 62-72), la galanterie instaure des rapports de genre inégalitaires, cette pratique signifiait aussi que les jeunes filles étaient reconnues comme ayant une individualité, une autonomie de choix, notamment dans la désignation de leur partenaire. Pour cela, les garçons devaient faire preuve de leur masculinité grâce à leurs talents de bons danseurs. Ils rivalisaient en galanterie, cadeaux, démonstrations sentimentales mais aussi en excentricités pour les séduire: "J'avais intégré la trompette à ma danse. Je m'accroupissais devant les filles» raconte Garrincha (Magnin 1998: 165). Une réécriture des codes de la masculinité s'opéra au travers de cette mode. Comme en fit l'expérience Penka Dembélé, la religion, la politique ou l'armée n'étaient plus des composantes du charisme masculin (Mann 2003: 75) aux yeux des jeunes filles:

«En 1963, j’ai fait mon service militaire et j’ai été nommé chef de l'orchestre qui a battu des records dans toute la région de Ségou. Un jour, pendant mon service, avec ma tenue militaire, jétais au bal à Kati. Je me suis fait planter successivement par deux filles en pleine danse. Elles ont déserté ma table et je me suis retrouvé tout seul avec mes trois limonades que j’avais payées. Mais par chance, le grand danseur Garrincha m'a reconnu et m’a supplié de monter sur scène pour jouer un morceau de biguine. Là ça a été magique, d'un seul coup, les filles m’ont entouré: "Un militaire qui joue comme ça! Bis, bis, bis!” Après ça tout le monde était à ma table!»

(Magnin 1998: 170)

Cette mode permettait donc aux jeunes urbains de se poser en groupe social unifié. Quels que fussent leur sexe, leur âge, leur origine géographique ou sociale, leur apparence corporelle était leur mode d'expression, illisible pour les non initiés. Si ce langage n'avait aucune teneur politique, le simple fait de se 
marginaliser de la communauté nationale, de se démarquer des «masses» devenait pourtant suspect au regard du pouvoir socialiste ${ }^{18}$.

\section{Le corps: enjeu des luttes idéologiques}

Tiraillés entre le projet socialiste et les aspirations des jeunes, les corps furent des espaces investis par le social et le politique. Les corps disciplinés par le travail ou l'organisation constituaient un des idéaux du régime ${ }^{19}$. Les images de propagande socialiste donnent à voir des corps mécanisés, que ce soit ceux des paysans courbés dans le travail de la terre, des soldats marchant d'un même pas, des chasseurs fusils à l'épaule ou des athlètes aux muscles saillants. Alors que l'État demandait à ces populations de se plier à une politique d'austérité, les jeunes affichaient, au travers de leurs vêtements, une attirance pour l'exubérance et la consommation. À l'opposé des postures rectilignes et uniformes, les jeunes urbains mirent en scène des corps désarticulés par la danse, dans des poses toujours plus originales:

«[ils] prenaient n'importe quelle position amusante et bizarre [...] les jeunes influencés par la musique sont excités, déchaînés, comme en transe, se sentent bien dans leur peau»

(Magnin 1998: 39).

Ces corps dynamiques, indomptés, tentaient de symboliser une certaine «fureur de vivre». Loin de la pudeur prônée par l'islam ${ }^{20}$, de l'obsession hygiéniste héritée de la période coloniale et de la vertu socialiste, les corps dévoilés, masculins et féminins, affichaient la sensualité, le plaisir, la jouissance. Les contacts physiques s'étalaient au grand jour comme l'explique Malick Sidibé:

«la jeunesse à cette époque a beaucoup aimé la musique twist, rock, afro-cubaine... car ça permettait aux garçons et aux filles de se rapprocher, de se toucher, de se coller. C'était impossible avec la musique traditionnelle»

(ibid.: 37).

Les bras le long du corps, le regard droit et provoquant, dans un déhanché accentué, les jeunes femmes de ses photographies apparaissent fières, impudiques et libres. Qu'elles fussent vêtues de robes gonflantes resserrées à la taille ou de minijupes découvrant leurs cuisses, ces jeunes femmes exhibaient et assumaient leurs corps. Les corps des jeunes hommes aussi étaient dénudés et érotisés: chemises ouvertes, torses et ventres offerts à la vue de tous, pantalons moulants mettant en valeur les fesses, cambrure prononcée... ces corps d'hommes longilignes, presque androgynes allaient à l'encontre des représentations viriles.

\section{La Révolution active: enfermement des corps et mise au pas de la jeunesse (1966-1968)}

En 1966, sentant la stabilité du régime menacée, une frange des dirigeants maliens radicalisèrent leur ligne politique. La peur naquit du coup d'État militaire 
qui renversa le régime socialiste ghanéen de N'Krumah, symbole du panafricanisme. Â l'intérieur du pays, la crise économique, la politique d'austérité et l'autoritarisme du régime ne faisaient qu'accroitre le mécontentement des populations, notamment rurales, à l'égard des dirigeants. S'appuyant sur l'organisation des jeunes et le syndicat des travailleurs, Modibo Keita déclencha une «Révolution active» en 1967, qui visait à purger l'appareil politique des «ennemis du socialisme» et à moraliser la société, selon le modèle de la Révolution culturelle chinoise 21 . L'une des cibles du pouvoir fut la «jeunesse marginale», étiquette qui s'appliquait à différents individus - chômeurs, petits délinquants, yéyés - pourvu qu'ils fussent jeunes. Ainsi, figures d'espoir dans les premières années de l'indépendance, les jeunes se transformèrent en menace pour la société car ils investissaient l'espace public, y affichaient leurs corps, leurs comportements, leurs sexualités et leurs plaisirs (Diouf 2003: 3).

\section{Mise en discours de la dégénérescence des corps et des esprits}

À lire les archives de police et de gendarmerie, la question du contrôle des populations jeunes fut au cœur des préoccupations étatiques dès 1960. Mais ce fut surtout à partir de 1965 que L'Essor publia une série d'articles criminalisant la jeunesse urbaine: délinquance juvénile, exode rural, vagabondage 22 et mendicité étaient amalgamés et érigés en problème de société. Les traces occidentales en matière de mode et de culture n'étaient cependant pas encore définies comme des symboles de la «crise juvénile». Ce fut le discours de Modibo Keita au centre d'apprentissage de Niaréla le 7 mai 1966 qui stigmatisa pour la première fois la jeunesse yéyé. Transgressive au départ, cette mode prit une teneur subversive lorsque l'État engagea une politique répressive à son encontre. Le discours sur la moralisation de la société s'accompagna d'une criminalisation de la jeunesse et de l'espace urbain, lieu de perdition, de prostitution et de délinquance. Dans sa lutte contre la «dépravation des mœurs», et quand il voulut dresser un portrait des adeptes de cette culture jeune, l'État rencontra beaucoup plus de difficultés à le faire pour les jeunes hommes que pour les jeunes filles.

Dans un premier temps, les garçons yéyés furent décrits comme des délinquants aux pratiques machistes: adeptes de la violence, amateurs de partenaires sexuelles multiples et consommateurs d'alcool. En 1966, L'Essor écrivait pourtant à leur sujet:

«Ils sont certes facteurs de beaucoup d'écarts, mais ils ont aussi une qualité que tout homme a $[\ldots]$ c'est le sens du combat $[\ldots]$ car tout homme est d'essence guerrière23».

L'enjeu n'était donc pas de réprimer ces jeunes mais plutôt de les éduquer, de les aider à canaliser leur combativité afin de la mettre au service de l'édification de la patrie. Mais, au fur et à mesure que le régime se durcit, les discours étatiques évoluèrent et les décrivirent comme des marginaux cherchant à déstabiliser l'ordre social. Au moment où Modibo Keita lançait la Révolution active en aout 1967, qui, mieux que ces jeunes, pouvait personnifier les «ennemis de 
l'intérieur», les «agents de l'impérialisme» dont le régime devait être purgé? La focalisation du pouvoir sur cette mode représentant «tout ce que l'occident a de laid et de décadent dans la phase finale de son système d'exploitation capitaliste ${ }^{24}$ relevait donc, avant tout, de la phraséologie révolutionnaire. Il serait néanmoins simpliste de réduire l'attitude de l'État à l'égard des yéyés à une seule instrumentalisation contre les intellectuels 25 . Comme en témoignent les stéréotypes véhiculés par le pouvoir à leur égard, les pratiques corporelles des jeunes urbains s'affrontaient directement au nouvel ordre moral socialiste:

«Les yéyés apparaissent sous la forme de cheveux très longs, une manière de se peigner et de s'habiller bizarre, entre autres: chemise débraillée ou attachée par le bas; pantalons taille basse à pattes d'éléphant, généralement multicolores [...] Ils se mettent souvent à crier comme des fous fieffés partout et à tout propos [...] Il n'est pas rare de voir certains d'eux esquisser des pas de singeries dans les rues $26 »$.

Aux yeux du pouvoir, ces attitudes corporelles masculines constituaient une rupture des codes gestuels socialement admis dans l'espace public. Dansant et chantant en public, mettant en avant leur nudité, affichant leurs relations amoureuses, ces jeunes ne respectaient pas la bienséance, la retenue requise pour des hommes face à leurs aînés:

«C'est la répudiation des règles de vie admises jusqu'ici, une révolte contre l'ordre établi27».

Pire, dans les discours de propagande, la réduction de ces jeunes à des corps étranges et sexualisés à outrance signifiait leur infériorité. Leur masculinité était niée dans ce contexte de Révolution active où la virilité était réaffirmée, où il était demandé aux pères de reprendre le contrôle des foyers pour instaurer la paix sociale: "La stabilité de la famille autour de l'autorité du père est sérieusement menacée 28 ». Seul l'ordre, le travail, la famille et une sexualité active, mais tournée vers la reproduction, constituaient les fondements de la masculinité socialiste. Ainsi, les pratiques sexuelles des jeunes urbains, axées sur le loisir et la jouissance immédiate, étaient jugées hors normes, voire monstrueuses. En témoigne la brutalité du vocabulaire employé pour les décrire qui faisait appel aux images les plus répulsives pour la population: «satyres, nymphomanes, forcenés, pervers, invertis pratiquant des séances orgiaques 29 ».

Mais les jeunes hommes n'étaient pas tant responsables de cette dépravation. Dans un Rapport de synthèse sur le problème de l'amoralité et de la licence chez la jeunesse publié en 1967, l'attrait des hommes maliens pour «la femme et les plaisirs y afférents» était décrit comme «atavique». Il était vain de lutter contre ce trait de caractère masculin, l'important était d'en limiter les excès car «la corruption sexuelle [...] altère en l'homme ce qu'il a de bien [...] le conduit au vol, au mensonge »30. Quels étaient donc les responsables de cette dépravation masculine? Tout simplement les référents culturels venus des pays étrangers et... les jeunes filles à la mode: 
«[ces] hordes de trotteuses [...] qui constituent en fait les principales actrices de leur propre malheur. Les minijupes et les décolletées sont portées en honneur chez elles. Ainsi elles exposent les attraits que la nature leur a donnés. Avec leur sens du raccourci en matière de réflexions, elles s'imaginent que la beauté tiendra surtout de la transformation artificielle de l'état initial 31 ».

Les mères étaient elles-mêmes accusées de favoriser «les relations de leurs filles avec des amants surtout, lorsque le profit matériel devient un complément au maigre salaire»; en d'autres termes, de les pousser à la prostitution. Cette rhétorique sur la monstruosité de la ville, dans laquelle l'ordre naturel des sexes était inversé, participait pleinement du discours du régime socialiste sur le retour à la terre. Sans parvenir pour autant à endiguer les migrations rurales (GaryTounkara 2003) et la contestation paysanne (Amselle 1978a), les dirigeants politiques érigeaient le monde rural comme symbole de cet «homme moral» 32 devant émerger de la nouvelle société socialiste. À l'inverse, la ville était cet espace où les femmes se transformaient en mauvaises mères, femmes abusives, tandis que leurs filles se muaient en corps «mutants", pour reprendre l'expression d'Elsa Dorlin (2006: 66), portant des stigmates virils par le seul fait de leur sexualité débordante et autonome 33 . Mais ces images de femmes frivoles, matérialistes et masculines apposées aux urbaines n'étaient pas le seul fait des discours étatiques. Pour de nombreux ruraux qui migraient temporairement sur Bamako lors de la saison sèche et qui y faisaient les yéyés - "À Bamako j'étais bien, je faisais le yéyé» (Amselle 1978b: 11) - il était impensable de se marier avec des urbaines qu'ils jugeaient trop «fantaisistes», "dépensières» et "paresseuses» (Meillassoux 1968: 77)34. En portant des vêtements sexy, les jeunes filles étaient accusées de vouloir soutirer de l'argent aux hommes, donc de les exploiter. Dans cette période de socialisme scientifique, les femmes furent érigées en symbole du capitalisme (Ivaska 2004: 104-121).

\section{Sexualisation de la répression}

La seconde étape de cette offensive contre la mode des jeunes urbains visa à la transformation physique des jeunes yéyés, premier pas, aux yeux de l'État, vers une reconversion morale. La presse lança des appels à la constitution, par les jeunes euxmêmes, de brigades de mœurs chargées de contrôler et de réprimer les marginaux:

«Qu'ensuite ils fassent des rondes là où il faut et quand il faut; alors ils procèderont sans coup ni blessures, aux opérations suivantes. Auparavant, ils seront armés d'une tondeuse et d'un lot de pagnes.

a) Immobilisation immédiate, partout où s'en présente l'occasion, de tout garçon porteur de cheveux "yéyé". La tondeuse passe au raz du cuir chevelu;

b) Immobilisation de toute jeune fille en minijupe ou dans tout autre accoutrement similaire. Résister aux équations, l'envelopper de deux pagnes et jeter dans la poubelle voisine la robe ou la minijupe yéyé froidement enlevée $35 »$. 
Un climat de peur s'installa sur Bamako. Les milices et les brigades, composées en grande partie de jeunes déclassés 36 , furent lancées à l'assaut d'autres jeunes: bastonnades, tortures, viols furent le lot courant des ennemis du socialisme:

«à l'époque c'était le yéyé. J'étais habillé comme ça et on m'a pris au marché. On m'a dit "pourquoi vous portez de telles tenues, c'est antirévolutionnaire" et ils m’ont conduit en prison. J'y ai passé une bonne journée, ils m’ont rasé la tête, sans mettre d'eau, à blanc... cela faisait très mal. (Les filles) on les maltraitait aussi, on les faisait crier, on violait je crois même ${ }^{37}$ ».

Si les archives restent muettes et que les témoins rencontrés demeurent encore très pudiques à l'égard des exactions commises à cette époque, il semble que les jeunes filles constituèrent des cibles privilégiées. Dans les rues, ces dernières devaient dorénavant faire profil bas et cacher leurs jupes sous de grands boubous. Lorsqu'elles se faisaient prendre dans une tenue jugée indécente, au mieux, leurs vêtements étaient déchirés et elles devaient payer une amende, au pire elles étaient conduites à la milice et violées. Cette répression remettait en cause l'indépendance acquise par les femmes dans les premières années du régime socialiste - circulation, travail, argent. Au-delà des violences physiques, un véritable encadrement des jeunes fut imposé : les élèves se virent obliger d'intégrer le Mouvement pionnier et de participer à des activités saines à la campagne: «sport, préparation militaire, $[. .$.$] chantiers { }^{38}$ ». Les comités scolaires étaient chargés de dénoncer leurs camarades yéyés afin de les faire comparaître devant un tribunal politique des mœurs, les jeunes chômeurs étaient envoyés dans les campagnes pour travailler. Un couvre-feu fut imposé aux mineurs à partir de 22 heures et les cinémas leur furent interdits s'ils n'étaient pas accompagnés d'un adulte. La Brigade des mœurs était autorisée à entrer dans les lieux publics comme privés pour traquer les couples adultères. Les miliciennes furent chargées d'inspecter les foyers afin d'en vérifier la propreté et des amendes furent infligées aux mauvaises ménagères, «les époux [étaient] épargnés, n'étant pas directement responsables de la situation ${ }^{39}$. Les grins de jeunes entrèrent temporairement en clandestinité, les soirées se privatisèrent et même Boubacar Traoré, l'icône yéyé, disparut des ondes de la radio nationale.

\section{La Dictature militaire:}

de l'insouciance à la politisation de la révolte (1968-1976)

En 1968, un coup d'État militaire renversa le régime de Modibo Keita. Dès le lendemain, la population sortit dans les rues de Bamako pour faire éclater sa joie. Un nouveau vent de liberté souffla, les clubs se reconstituèrent, les jeunes réoccupèrent l'espace public et réaffirmèrent leur identité à la faveur de la mode, comme s'en aperçut rapidement la gendarmerie de Bamako: 
«Il est à noter que dans ce dernier trimestre la carence de la Brigade des Mœurs a donné un nouveau souffle au comportement indécent des jeunes pratiquant (le Yéyéisme, Hypisme et Thé club) 40 ».

\section{Vers une libération sexuelle?}

Le contexte des années 1970 était cependant bien différent de celui des indépendances. Le Mali était frappé par une crise économique résultant des échecs du régime socialiste, du contexte international, de la sécheresse de 1972. Les loisirs prirent de plus en plus de place dans les occupations de la jeunesse, mais leur accessibilité instaura une frontière entre jeunesse travailleuse et jeunesse scolaire. Pour les premiers, il s'agissait essentiellement de réseaux de solidarité masculins où l'on discutait de la vie mais aussi de politique au grand dam des autorités militaires qui, dans la continuité du régime socialiste, criminalisèrent ces «thés clubs»:

"Chaque membre est tenu de contribuer ou matériellement, ou financièrement, il en résulte que certains volent du thé, du sucre et de l'argent à leurs parents au profit de l'association. Or, on dit qui vole un œuf, vole un bœuf. L'habitude étant une seconde nature et le besoin toujours croissant, ils finiront par glisser sur la mauvaise pente ${ }^{41 »}$

(Nédélec 1994: 398).

Ce fut autour des pratiques corporelles des jeunes scolarisés que se cristallisa l'aspiration à une liberté de mouvements et d'allures. Au moment des grandes chaleurs de mars à juin, ces derniers se retrouvaient le dimanche au bord du fleuve Niger à la Chaussée et au Rocher aux aigrettes ${ }^{42}$ pour se baigner. Selon Yousouf Doumbia dit Garrincha, sous couvert de jeux, l'eau devint un espace où les corps dénudés entraient en contact, où la sexualité se libérait:

«Dès le jeudi, au grin on préparait cette sortie au bord du fleuve Niger avec les clubs Trianon et les "On s'en fout". On se cotisait pour acheter le mouton et les boissons. Les filles apportaient leur linge dans les calebasses, on y allait à pied ou à moto. J'apportais mon Teppaz à piles, mes 45 tours et on commençait la fête jusqu'à 20 heures. On se baignait, on se chamaillait, on dansait [...] On flirtait, on se touchait sous l'eau, on s'embrassait et on raccompagnait les filles avant la nuit»

(Magnin 1998: 167).

Symbole de ce temps du corps à corps, le désir était dévoilé au grand jour. Malick Sidibé photographiait des corps enlacés sur la plage, sur un lit, des baisers langoureux. Alors que la mode des seins nus fut une provocation contre le puritanisme en Europe et constitua un symbole d'émancipation sexuelle, il n'y avait rien d'extraordinaire pour une Malienne d'être en pagne, la poitrine dénudée au bord du Niger. L'adoption du bikini par les jeunes filles était bien plus subversive. Comme l'exprime Jean Allman (2004: 6), la mode peut être un langage parlé n'importe où, mais elle n'est jamais une langue universelle: dans les 
années 1970, les jeunes Maliennes s'emparèrent du soutien gorge, exhibèrent cet attribut de mode doté d'une signification émancipatrice, tandis que les Françaises s'en débarrassaient. Il est cependant difficile de définir la teneur libératrice de ces corps féminins dénudés qui oscillaient entre une sexualité décomplexée et une soumission aux désirs masculins. Sur les clichés de Malick Sidibé, de plus en plus de jeunes filles reproduisaient les poses des mannequins vues dans les magazines féminins français: corps allongés, lascifs, s'offrant à l'amour, corps soumis. Le nouvel idéal féminin qui prenait forme était celui de femmes séductrices, sujets et objets de consommation. Quant aux jeunes hommes, ils posaient en conquérants - torses bombés, seuls au-dessus d'un rocher - en protecteurs enlaçant les filles. Ainsi, si les attitudes corporelles constituèrent une forme de langage, il demeurait plus facile pour ces jeunes de montrer des corps dénudés que de parler de nudité: la libération des corps ne s'accompagna pas d'une libération de la parole - notamment féminine - sur la sexualité.

\section{Quand la mode se politise...}

Pour marquer leur opposition au régime déchu, les militaires mirent fin aux organisations de jeunes et interdirent de parler de politique. Prenant le contre-pied, une partie de la jeunesse se tourna vers une mode rock davantage influencée par le mouvement noir américain et bien plus politisée que celle des années soixante:

«Nous portions des chemises à fleurs et des signes de paix; nous fumions de la marijuana; nous étions contre la guerre du Vietnam et contre l'apartheid en Afrique du Sud; nous étions pour le Black Power, les Black Panthers et les Black Muslims aux États-Unis»

explique Manthia Diawara (2001: 120) surnommé «J. B.» en référence à James Brown et membre du groupe «les Rockers». Georges Jackson, Angela Davis, Muhammad Ali, Eldridge Cleaver, Malcom X et Jimi Hendrix constituaient les nouvelles icônes auxquelles il fallait s'identifier. Arborant leurs coupes afro, parés de lunettes noires, vêtus de pantalons pattes d'éléphant, ces jeunes se déplaçaient en groupe sur leurs talons compensés et faisaient semblant de ne pas parler français. Ils se considéraient en lutte contre le racisme et s'approprièrent les référents culturels guerriers. Certains groupes se faisaient appelés «les Sofas» - cavalier en bambara - «les Tondjons» - les fantassins. Lors d'un «Woodstock à Bamako » organisé à la Maison des anciens combattants en 1971 par Manthia Diawara et son ami «Sly» - en référence au groupe Sly and The Family Stone les tenues hippies, kitch, rock et rétro rivalisèrent avec les costumes traditionnels de chasseurs ${ }^{43}$. Quelques jeunes filles s'emparèrent du pantalon et en firent un symbole de leur appartenance à cette mode masculine, rebelle, étudiante, donc largement minoritaire. Cette mode s'inscrivait dans un contexte où la contestation gagnait de plus en plus les lycées et les universités. Si les étudiants supportèrent très mal l'autoritarisme et les exactions des milices sous Modibo Keita, ils 
virent d'un plus mauvais œil encore l'installation d'un régime militaire. Ainsi, dès 1969, les étudiants de l'École normale supérieure s'organisèrent au sein de l'ADEEN Sup ${ }^{4}$ et engagèrent une grève qui fut violement réprimée. Progressivement, les clubs disparurent et furent remplacés par des associations scolaires qui déclenchèrent une succession de grèves de 1976 jusqu'au début des années 1980 (Nédélec 1993).

Indépendance cha-cha, chanson composée par Joseph Kabasele en 1960, ode à la liberté, symbole d'espoir, ce tube de la rumba congolaise fit le tour de l'Afrique francophone. Pour la jeunesse urbaine de Bamako, il s'agissait bien de vivre et d'expérimenter cette indépendance nouvellement acquise, et non d'intégrer les carcans d'un système monolithique et autoritaire. Plus romantique que politique, cette rébellion s'exprimait par l'adoption d'un mode de vie non conforme à la morale en vigueur. En réaction à l'uniformisation des corps imposée dans les organisations du régime socialiste, cette jeunesse s'appropria une mode cosmopolite pour revendiquer son autonomie par rapport à l'autorité étatique et parentale, en tant que jeunes, mais aussi en tant que femmes, et en tant qu'hommes. Le corps, au travers de ses apparences, devint un terrain d'exploration et d'expérimentation de pratiques d'individualisation plurielles: de sexe, d'âge, de classe. Si la domination masculine - notamment maritale - ne fut pas remise en cause, la façon dont les corps furent sexués transgressa, parfois, les frontières de genre: corps longilignes et dénudés pour les hommes, cheveux courts et cigarette à la bouche pour les filles. Pour reprendre les mots de Florence Rochefort «des usages rebelles ont une portée politique même s'ils ne s'inscrivent pas d'emblée dans une vision théorique cohérente et intellectualisée du corps. Ils précèdent le mouvement de politisation du social et du privé, les nourrissent et s'en trouvent transformés en retour» (2008: 615-616). L'État malien ne fut pas dupe et chercha rapidement à reprendre en main ces corps «indociles» (Mbembe 1988: 219) porteurs d'un message qui transcendait l'euphorie des indépendances. Ces corps devinrent un symbole de plus en plus prégnant du bras de fer mené entre le pouvoir militaire et les jeunes: hommes tondus, soumis aux exercices militaires et aux coups; femmes déshabillées de force, cheveux rasés et menacées de viol, furent les techniques employées par les autorités pour tenter de faire taire la contestation des scolaires et des étudiants et étudiantes, jusqu'à la chute de la dictature militaire en 1991.

Thématique futile au premier abord, l'étude de la mode permet d'appréhender des dynamiques sociales souterraines et de complexifier l'analyse des rapports de force qui se jouèrent au lendemain des indépendances. Au-delà des discours d'homogénéisation de la jeunesse produits par le Parti unique, cette dernière se révèle protéiforme: militante, milicienne, travailleuse, yéyé... La jeunesse bamakoise de ces années se décline en une multitude de catégories qui 
pouvaient se superposer ou s'affronter violement. Si l'accent a été mis ici sur la répression subie par les jeunes à la mode, il ne faut pas oublier que l'ensemble de la population endura les exactions des miliciens, et miliciennes, chargés du contrôle social: maris emprisonnés, femmes violées, commerçants dépossédés de leurs marchandises, anciens roués de coups en public. La déstabilisation de l'ordre social ne fut donc pas le seul fait de la jeunesse «à la mode». Cette accentuation progressive du pouvoir de la milice au moment de la Révolution active accéléra, par ailleurs, la chute du régime socialiste et la prise du pouvoir par un autre groupe de jeunes, militaires cette fois. Ainsi, développant toute une gamme de registres d'énonciation et de stratégies d'action, les jeunes furent au cœur des luttes sociales et politiques des années 1960-1970.

\section{Ouvrages cités}

Allman, Jean. 2004. Fashioning Africa. Power and the Politics of Dress. Bloomington, Indiana University Press.

AMSELLE, Jean-Louis.1978a. «La conscience paysanne: la révolte de Ouolossébougou (juin 1968, Mali)", Revue canadienne des études africaines, vol. 12, $\mathrm{n}^{\circ} 3: 339-355$.

- 1978b. «Migrations et sociétés néo-traditionnelles: le cas des Bambara du Jitumu (Mali)», Cabiers d'études africaines, vol. 18, n 72: 487-502.

AYINA, Egbomi. 1987. «Pagnes et politique", Politique africaine, n 27: 47-54.

BARTHÉLÉMY, Pascale. 2004. «Femmes, africaines et diplômées: une élite auxiliaire à l'époque coloniale. Sages-femmes et institutrices en Afrique occidentale française (1918-1957)», thèse d'histoire, université de Paris VII-Denis Diderot. — et Jean-Hervé JÉZÉQUEL. 2007. «Marier les "demoiselles frigidaires" et les "mangeurs de craies": l'idéal du ménage lettré et l'administration coloniale en Afrique Occidentale Française (AOF)», in Odile Goerg (éd.), Perspectives historiques sur le genre en Afrique. Paris, L'Harmattan (Cahiers Afrique): 77-96.

BARTHES, Roland. 2009. [1980] La chambre claire. Note sur la photographie. Paris, Seuil (Cahiers du cinéma).
BAYART, Jean François. 1979. L'État au Cameroun. Paris, Presses de la Fondation nationale des sciences politiques.

Comaroff, Jean et John Comaroff. 2000. «Réflexions sur la jeunesse. Du passé à la postcolonie», Politique africaine, no 80: 90-110.

Diallo, Assitan. 2004. «Paradoxes of Female Sexuality in Mali», in Signe Arnfred, Re-thinking Sexualities in Africa. Uppsala, Nordiska Afrikainstitutet: 173-189.

Diawara, Manthia. 2001 [1998]. En quête d'Afrique. Paris-Dakar, Présence africaine (éd. orig., In Search of Africa. Cambridge, Harvard University Press).

Diouf, Mamadou. 2003. «Engaging Postcolonial Cultures: African Youth and Public Space», African Studies Review, $\mathrm{n}^{\circ} 2: 1-12$.

DoRLIN, Elsa. 2006. La matrice de la race. Généalogie sexuelle et coloniale de la nation française. Paris, La Découverte (Textes à l'appui).

FitZPATRICK, Sheila. 2006. «Social Parasites. How Tramps, Idle Youth, and Busy Entrepreneurs Impeded the Soviet March to Communism», Cahiers du monde russe, $\mathrm{n}^{\circ} 1-2$ : 377-408.

Gandoulou, Justin-Daniel. 1989.

Dandies à Bacongo. Le culte de l'élégance 
dans la société congolaise contemporaine. Paris, L'Harmattan (Logiques sociales).

GARY-TOUnKara, Daouda. 2003. "Quand les migrants demandent la route, Modibo Keita rétorque: "Retournez à la terre!”. Les Baragnini et la désertion du "Chantier National" (1958-1968)», Mande Studies, n 5 : 49-64.

GoERG, Odile. 2007. «Femmes adultères, hommes voleurs. La "justice indigène" en Guinée», Cabiers d'études africaines, $n^{\circ}$ 187-188: 495-522.

GoFFMAN, Erving. 2002. [1977] L'arrangement des sexes. Paris, La Dispute (Le genre du monde), (éd. orig. "The Arrangement between the Sexes", Theory and Society, vol. 4, n 3: 301-331).

Gondola, Charles-Didier. 1997. «Oh, rio-Ma! Musique et guerre des sexes à Kinshasa, 1930-1990 ", Revue française d'histoire d'outre-mer, vol. 84, n' 314: 51-81.

HoвsBawm, Eric J. 1999. [1994] L'âge des extrêmes. Le court vingtième siècle, 1914-1991. Bruxelles, Complexe (Bibliothèque Complexe), (éd. orig., Age of Extremes. The Short Twentieth Century, 1914-1991.

Londres, Michael Joseph).

IVASKA, Andrew M. 2004. «Anti-mini Militants Meet Modern Misses: Urban Styles, Gender and The Politics of National Culture in 1960s Dar es Salam, Tanzania", in Jean Allman (éd.), Fashioning Africa: Power and The Politics of Dress. Bloomington, Indiana University Press: 104-121.

JÉZÉQUEL, Jean-Hervé. 2002. «Les “mangeurs de craies”. Les instituteurs diplômés de l'école normale William Ponty. Sociohistoire d'une catégorie professionnelle à l'époque coloniale (1900-1960)», thèse d'histoire, École des hautes études en sciences sociales, Paris.

- 2007. «Grammaire de la distinction coloniale. L'organisation des cadres de l'enseignement en Afrique occidentale française (1903-fin des années 1930)", Genèses, nº 69: 4-25.

Konaré, Adam Ba. 1993. Dictionnaires des femmes célèbres du Mali. Bamako, Jamana.
LIEVE, Joris. 2002 [1997]. Mali Blues. Je chanterai pour toi. Arles, Actes Sud (Babel), (éd. orig., Mali blues. En andere verhalen. Amsterdam, Meulenhoff).

Magnin, André. 1998. Malick Sidibé. Zurich, Scalo Verlag.

MANN, Gregory. 2003. «Old Soldiers, Young Men: Masculinity, Islam, and Military Veterans in Late 1950s Soudan Français (Mali)», in Lisa A. Lindsay and Stephan F. Miescher (éd.), Men and Masculinities in Modern Africa. Portsmouth, Heinemann: 69-85.

Mвемвe, Achille. 1988. Afriques indociles. Christianisme, pouvoir et État en société postcoloniale. Paris, Karthala (Chrétiens en liberté).

MeILlassoux, Claude. 1968. Urbanization of an African Community. Voluntary Associations in Bamako. Seattle, University of Washington Press (American Ethnological Society).

Morin, Edgar. 1969. «Culture adolescente et révolte étudiante», Annales. Économies, sociétés et civilisations, vol. 24, $\mathrm{n}^{\circ} 3$ : 765-776.

NÉDÉLEC, Serge. 1993. «La révolte scolaire malienne de 1979-1980: essai d'histoire immédiate», in Catherine Coquery-Vidrovitch (éd.), Histoire africaine du XXe siècle. Sociétés, villes, cultures. Paris, L'Harmattan: 19-46.

— 1994. «Jeunesses, sociétés et État au Mali au XXe siècle», thèse nouveau régime d'histoire, université de Paris VII.

NiMIs, Erika. 2005. «De Nadar à Keita: une histoire franco-malienne de la photographie», in GEMDEV et Université du Mali, Mali-France. Regards sur une histoire partagée [Colloque «Regards croisés France-Mali», Bamako, janvier 2005]. Paris, Karthala (Hommes et sociétés): 381-403.

Poiger, Uta G. 2000. Jazz, Rock, and Rebels. Cold War Politics and American Culture in a Divided Germany. Berkeley, University of California Press (Studies on the History of Society and Culture).

ROCHEFORT, Florence. 2008. «La politisation des corps", in Philippe Artières et Michelle Zacharini-Fournel (éd.), 68. Une histoire collective, 1962-1981. Paris, La Découverte: 615-621. 
RoNDEAU, Chantal. 1994. Les paysannes

du Mali. Espaces de liberté et changements.

Paris, Karthala (Hommes et sociétés).

VeIllon, Dominique, Valérie Guillaume

et Michèle Ruffat. 2000.

«Essai Bibliographique. La mode

des années 1960 ", Bulletin de l'Institut

d'bistoire du temps présent, $\mathrm{n}^{\circ} 76$,

http://www.ihtp.cnrs.fr/spip.php\%3Frubrique

86.html (consulté le 8 septembre 2010).

Werner, Jean-François. 1996.

«Seydou Keïta (Catalogue de l'exposition des photographies présentées à l'occasion de l'exposition "Seydou Keïta" du 28 octobre au 27 novembre 1994); Malick Sidibé.

Bamako 1962-1976. (Catalogue des photographies présentées à l'occasion de l'exposition "Malick Sidibé" du 30 avril au 11 juin 1995); Mama Casset et les précurseurs de la photographie au Sénégal, 1950 », Cahiers d'études africaines, vol. $36, \mathrm{n}^{\circ} 141$ : 313-316.

\section{Notes}

* Une première version de ce texte fut discutée à la journée d'étude «Le corps et ses genres. Les dimensions corporelles des différences sexuées» du 6 juin 2009, université de Paris I. Je remercie particulièrement Sophie Poirot-Delpech, Pascal Ory et Johanna Siméant pour leurs commentaires.

1. Expressions employées par Modibo Keita dans ses discours: 1967. L'An I de la Révolution, Imprimerie de Koulouba: 210.

2. L'Essor, «Le sens du Concours du plus beau bébé», 2 août 1966: 1-3.

3. La définition que Jean et John Comaroff donnent de ces cultures du XXIe siècle fait écho aux dynamiques culturelles maliennes des années 19601970: «cultures du désir, de l'expression de soi et de la représentation - ainsi que l'affirmation, en certains lieux, de formes de politisation puissantes, quoique non conventionnelles» (2000: 94).

4. Quelques exemples: le Super Biton de Ségou créé en 1960 et le Super Rail Band, orchestre du buffet-hôtel de la gare de Bamako, créé en 1970 au sein duquel Salif Keita et Mory Kanté firent leurs premiers pas.

5. Pour l'historien Eric Hobsbawm, dans les années 1960 «une culture mondiale de la jeunesse était née» et «cette culture jeune devint la matrice de la révolution culturelle».
6. Informations provenant d'entretiens réalisés en décembre 2009. Nombre d'étudiants maliens allaient faire leurs études à Dakar et à Abidjan. Les cheminots et les commerçants furent aussi des vecteurs de diffusion de cette mode.

7. À ce sujet voir le film de Moustapha Alassane, $L e$ retour d'un aventurier, Niger, 1966, et le reportage de Serge Moati, Les Cow-Boys sont Noirs, Niger, 1966.

8. Nombre des acteurs de ces clubs des années 1960-1970 sont aujourd'hui passés au statut d'aînés sociaux. La respectabilité venant avec l'âge, ils se posent en chefs de famille, fervents musulmans et reprennent, à l'encontre de la nouvelle génération, les discours sur la dépravation morale de la jeunesse qui leur étaient alors adressés. D'où la difficulté de mener des entretiens approfondis sur ces questions, notamment avec les femmes. Certaines personnes demandent à Malick Sidibé de ne pas publier, voire de détruire, les clichés de leur jeunesse.

9. Union soudanaise-Rassemblement démocratique africain. Parti unique dirigé par Modibo Keita jusqu'en 1968.

10. Expression utilisée dans la presse et les rapports étatiques pour qualifier la mode yéyé. La connotation très péjorative de ce terme, synonyme de délinquance, demeure dans les mémoires collectives. Dans les entretiens réalisés, les témoins 
qui reconnaissent avoir suivi cette mode, rejettent cependant le qualificatif de "yéyé» qu'ils assimilent aux «mauvais garçons».

11. Plusieurs études permettent d'appréhender les normes sociales, notamment genrées, à l'œuvre dans la société malienne contemporaine (Diallo 2004; Rondeau 1994: 362). Au regard de l'hétérogénéité des pratiques sociales et culturelles présentent sur le territoire, il serait périlleux et réducteur de dresser ici une vue d'ensemble de ces normes.

12. Archives nationales du Mali (par la suite ANM), Fonds de l'US-RDA BPN, 146e carton: 568 Milice Populaire 1964-1968, «Lettre de la Secrétaire permanente de la Commission sociale des Femmes au Directeur de la Milice populaire du Mali», Bamako, 15 novembre 1967.

13. Dans sa thèse sur la jeunesse malienne, Serge Nédélec (1994) date de 1962 le début des désillusions à l'égard des organisations de jeunesse.

14. Le terme "évolué» appartient au vocabulaire colonial. Il désignait les Africains formés à l'école coloniale, ayant intégrés les habitus occidentaux et qui constituèrent les auxiliaires lettrés de l'administration. Pour une analyse des catégories coloniales appliquées aux fonctionnaires africains, voir Jézéquel (2007).

15. Né en 1935, tailleur, tient son surnom d'un footballeur brésilien.

16. Valorisation du modèle conjugal occidental (division sexuée des tâches au sein du foyer, mariage civil librement consenti par les époux et monogame, romantisme), intéressement des jeunes hommes à la gestion du foyer (Jézéquel 2002: 792) et «formation intellectuelle et morale virile» pour les filles (Barthélémy 2004: 162).

17. Sans doute en raison d'un contrôle social de leur apparence plus intense.

18. La construction nationale était fondée sur l'uniformité comme en témoigne la devise malienne «Un Peuple. Un But. Une Foi» et les slogans scandés lors de la Révolution active de 1967, « un seul Parti: l'USRDA, un seul guide: Modibo Keita, une seule option: le socialisme». Toute tentative de différentiation était perçue comme subversive.

19. Peut-on voir là une continuité avec le «dressage des corps» caractéristique de l'école normale William Ponty sous la colonisation, là même où furent formés les leaders nationalistes maliens comme Modibo Keita (Jézéquel 2002: chap. x)?

20. Seule une étude approfondie permettrait d'évaluer la dimension normative de l'islam. Quasiment absent des discours politiques et de ceux des jeunes étudiés, ce référent religieux était cependant utilisé par les parents pour critiquer la politique socialiste, tout comme le comportement de leurs enfants.

21. À la grande différence de la Chine que cette «révolution» ne fit, officiellement, aucun mort.

22. Le Code pénal définit le vagabondage comme un délit puni de quinze jours à six mois d'emprisonnement et d'interdiction de séjour de deux à cinq ans en cas de récidive. "Le travail est un devoir pour tout Malien. En conséquent, le vagabondage est un délit», loi n 61-99 AN-RM du 3 aout 1961, art.145.

23. L'Essor, «Pourquoi sont-ils Yé-Yé?», 22 juin 1966: 3-4.

24. ANM, Fonds de l'US-RDA BPN, 110e carton: 420 Rapport de synthèse sur le problème de la moralité et de la licence chez la jeunesse, 1967: 13.

25. Au cours de la Révolution active, une série de stéréotypes sociaux furent énoncés par le pouvoir: les paysans comme symboles du prolétariat et les cadres, les fonctionnaires, les étudiants, comme bastions de la contre-révolution.

26. L'Essor, «La Crise juvénile au Mali a été le thème d'une Conférence à l'ENS de Badalabougou ", 4 mai 1967: 1-4.

27. ANM, Fonds de l'US-RDA BPN, 110e carton: 420 Rapport de synthèse sur le problème de la moralité et de la licence chez la jeunesse, 1967: 13.

28. Ibid.

29. L'Essor, «À propos de la crise juvénile au Mali», éditorial, 18 mai 1967: 1-4.

30. ANM, Fonds de l'US-RDA BPN, 110e carton: 420 Rapport de synthèse sur le problème de la moralité et de la licence chez la jeunesse, 1967: 13. La production de ce rapport mobilisa les plus hautes instances de l'État: les commissions (politique, sociale et culturelle, administrative et judiciaire) du Bureau politique, les ministères (Justice, Éducation nationale, Santé publique et Affaires sociales), la Commission nationale de la jeunesse ainsi que les comités politiques des Grandes Écoles nationales.

31. L'Essor, «La Crise juvénile (suite)», 6 mai 1967: 1-3.

32. L'Essor, «Liberté n'est pas synonyme d'extravagance», 15 mars 1967: 1-2.

33. En opposition à une féminité morale et maternelle prônée par la propagande socialiste.

34. «On the other hand, they praise the submissiveness, fidelity and working capacity of country girls», écrit Claude Meillassoux. 
35. L'Essor, "Première mesure à prendre dans la lutte contre la crise juvénile», 19 mai 1967: 1. Nous soulignons.

36. L'idée selon laquelle les miliciens étaient recrutés parmi les jeunes déscolarisés et chômeurs mériterait d'être d'avantage étudiée et nuancée: elle fut véhiculée après le coup d'État de 1968 et appartient toujours aux représentations des Bamakois.

37. Entretien réalisé à Kati, mars 2010, avec Cheick Oumar Fofana, dix-huit ans, lycéen, actif au sein de l'organisation de jeunesse du Parti en 1967.

38. ANM, Fonds de l'US-RDA BPN, 110e carton: 420 Rapport de synthèse sur le problème de la moralité et de la licence chez la jeunesse, 1967: 13.

39. ANM, Fonds de l'US-RDA BPN, 55 e carton: 144 Commission sociale des femmes, lettre de la commission sociale des femmes au Médecin chef du Service d'hygiène de Bamako, 5 août 1967: 1.

40. Archives du ministère de l'Administration territoriale et des Collectivités locales (AMATCL), Carton N2/1-2, Dossier N2/1-2: Rapports trimestriels de la Gendarmerie. 1970-1971-1972. Confidentiel, $\mathrm{n}^{\circ} 121 / 4$, Rapport du $2^{e}$ trimestre 1970 de la Compagnie de Bamako.

41. IGS, DAD, La délinquance juvénile au Mali, rapport 1974 : 13. Cité par Serge Nédélec.

42. Plages situées en périphérie de Bamako.

43. Le photographe Mamadou M'Bay couvrit cet événement mais malheureusement ses photographies ne sont encore ni éditées, ni diffusés.

44. Association des étudiants de l'École normale supérieure. 